\title{
'Bath-plug' technique for the endoscopic management of cerebrospinal fluid leaks
}

\author{
P. J. Wormald, F.C.S.(S.A.), F.R.C.S.(Ed.)*, Mike McDonogh, F.C.S.(S.A.)
}

\begin{abstract}
In the past cerebrospinal fluid (CSF) leaks were managed via a craniotomy with intradural repair. With the advent of endoscopic sinus surgery, transnasal repair has become more popular. The comparatively low morbidity and high success rate of endoscopic repair has made it the treatment of choice for CSF leak repair. As more surgeons practice endoscopic sinus surgery, the incidence of iatrogenic CSF leaks has increased. It is important that endoscopic sinus surgeons be aware of the techniques for closing such leaks. The aim of this study is to present a new technique for the endoscopic repair of CSF leaks. Since the technique was devised the authors have used the technique on six patients. All patients had lumbar drains inserted and five patients had intra-thecal fluorescein inserted for location of the leak. All repairs have been successful with an average follow-up of 13 months. The technique consists of introducing a fat plug with a specifically secured vicryl suture into the intradural space and placing traction on the suture to seal the defect much as a bath plug seals a bath. There were no complications in any of the patients either intra- or post-operatively. The difficulties and possible complications of the technique as well as the use of a lumbar drain and intra-thecal fluorescein are discussed.
\end{abstract}

Key words: Cerebrospinal rhinorrhoea; Endoscopy, surgery

\section{Introduction}

The popularity of endoscopic sinus surgery has surged in the last decade with most rhinologists considering it the management of choice for chronic sinusitis resistant to medical therapy and nasal polyposis (Lund and MacKay, 1994). One of the major complications of endoscopic sinus surgery is the development of a cerebrospinal fluid (CSF) leak (incidence 0.9 per cent) (Stankiewicz, 1991). As an accepted complication of endoscopic sinus surgery, the surgeon should be capable of managing such a problem if faced with it. However, as the endoscopic management of CSF leaks is relatively new, a number of different techniques have been used to close the leak (Papay et al., 1989; Mattox and Kennedy, 1990; Stankiewicz, 1991; Dodson et al., 1994; Hoa, 1996; Hughes, et al., 1997). Traditionally CSF leaks have been managed by the neurosurgeons via a frontal craniotomy. This conventional approach has a success rate of between 60 and 80 per cent (Aarabi and Leibrock, 1992; Hughes et al., 1997) but is also associated with significant morbidity from frontal lobe retraction and anosmia (Carmel and Komisar, 1991; Dodson et al., 1994; Hisamatsu and Ganbo, 1995). The success rate of endoscopic closure of spontaneous and traumatic CSF leaks of the anterior cranial fossa is between
76 per cent (Dodson et al., 1994) and 100 per cent (Mattox and Kennedy, 1990) with the majority of series in the region of 90 per cent (Papay et al., 1989; Hoa, 1996; Hughes et al., 1997). As it has minimal morbidity, preservation of the sense of smell and a high success rate, endoscopic closure is now recognized as the treatment of choice (Mattox and Kennedy, 1990; Hughes et al., 1997).

Methods of endoscopic CSF leak closure vary. However, most authors agree that once the defect is identified, the mucosa around the defect should be removed in order to allow the graft to firmly adhere to the skull base (Mattox and Kennedy, 1990; Dodson et al., 1994; Hoa, 1996). Varying materials have been used to close the fistula. The most common being free mucosal graft, free turbinate mucosa, fascia lata, temporalis fascia or abdominal fat (Mattox and Kennedy, 1990; Stankiewicz, 1991; Dodson et al., 1994; Hughes et al., 1997). These grafts were supported by a combination of muscle, fat and Gelfoam $^{\circledR}$. One of the problems with the techniques described thus far is that the graft is placed on the undersurface of the dural defect and may be pushed away from the dural tear if the CSF pressure increases, with subsequent recurrence of the leak. The aim of this paper is to describe a surgical technique that overcomes this problem.

From the Division of Otolaryngology*, Prince of Wales Hospital, Hong Kong. Accepted for publication: 19 August 1997. 

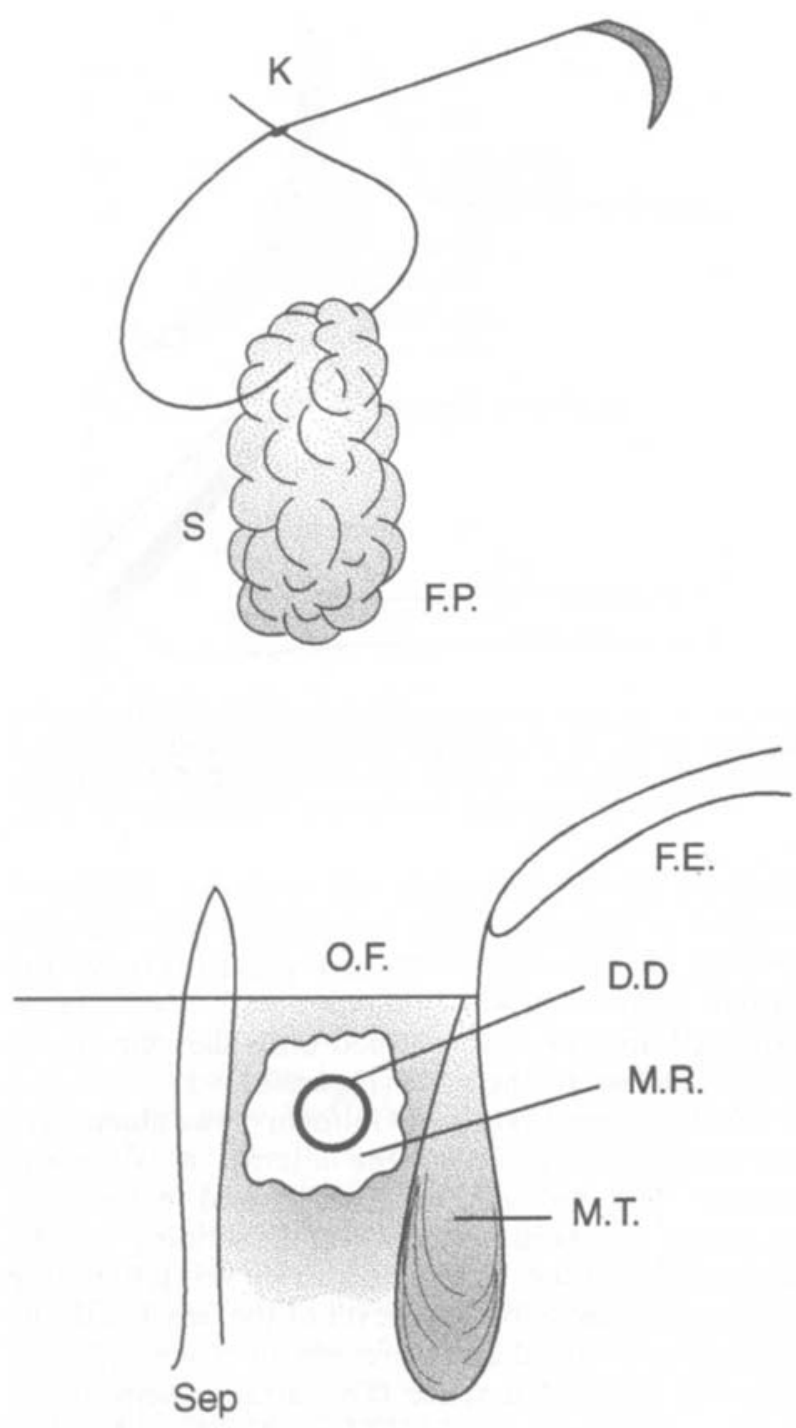

FIG. 1

Fat plug with knot being tied with a four 0 vicryl suture. Coronal section through the anterior cribriform plate with dural defect illustrating removal of surrounding mucosa for about $5 \mathrm{~mm}$. Key: $\mathrm{DD}=$ dural defect, $\mathrm{FE}=$ fovea ethmoidalis, $\mathrm{FP}=$ fatplug, $\mathrm{K}=$ knot, $\mathrm{MR}=$ mucosa removed, $\mathrm{MT}=$ middle turbinate, $\mathrm{OF}=$ olfactory fossa, $\mathrm{S}=$ four 0 vicryl suture, sep $=$ septum.

\section{Materials and methods}

\section{Technique}

The key to successful management of CSF leaks is the precise identification of the site of the dural tear. All patients underwent endoscopic nasal examination and in some cases the site of the CSF leak was apparent. If possible our patients had CSF collected and tested for sugar and $\beta$ transferrin. Highresolution $2 \mathrm{~mm} C T$ scan of the sinuses was performed and in some cases, a magnetic resonance image (MRI) was performed. At the time of surgery, all patients had a lumbar drain inserted. CSF was taken for microscopy and culture. If the site of the leak was still uncertain (five of the six patients), $10 \mathrm{ml}$ of CSF was withdrawn and mixed with between $0.25-0.4 \mathrm{ml}$ five per cent fluorescein. This mixture was injected into the intra-thecal space, the lumbar drain clamped and the patient placed in the head-down position to allow diffusion of the fluorescein throughout the CSF. The site of the CSF leak was visualized and if fluorescein had been used, the CSF appeared a yellowish-green colour.

In two patients with meningo-encephalocoeles, the brain and meninges were amputated flush with the skull base. Bipolar diathermy was used for haemostasis. In all cases the mucosa around the fistula was carefully dissected away from the bony defect for approximately $5 \mathrm{~mm}$. A free mucosal graft was harvested from the middle turbinate or lateral nasal wall and placed in saline. Fat was harvested from the ear lobe or from a peri-umbilical incision on the abdomen. The fat plug was approximately $1 \mathrm{~cm}$ in length and at least double the width of the dural defect. A four 0 vicryl stitch was placed and knotted in the one end of the fat plug (Figure 1). Approximately $5 \mathrm{~mm}$ of mucosa around the defect was gently removed (Figure 1). The suture was then passed from the knot down the length of the fat plug (Figure 2). The fat plug was covered with slow setting Tisseal ${ }^{\circledR}$ fibrin glue. The knot and fat plug were placed with a paediatric Blakesley forceps in

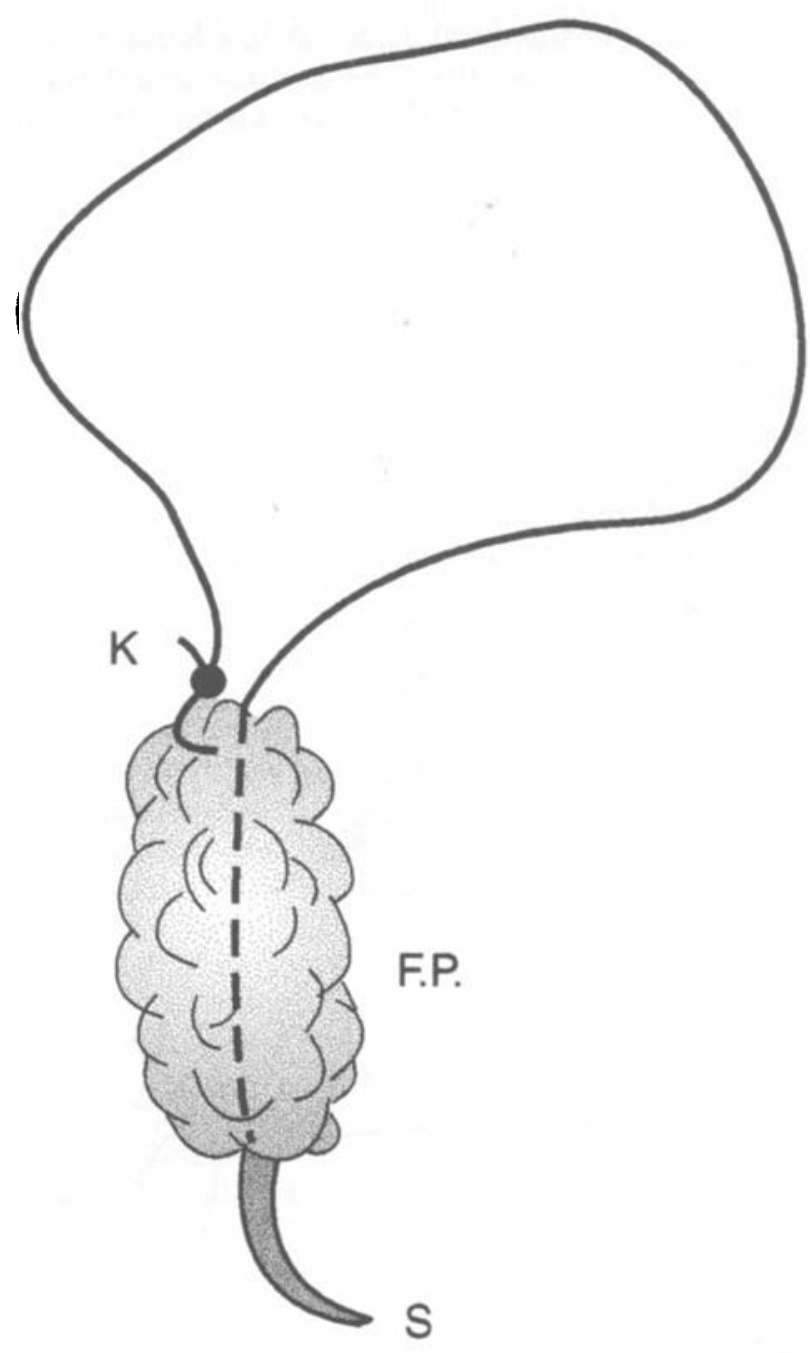

FIG. 2

The vicryl suture is passed down the length of the fat plug. Key: FP fat plug, $\mathrm{K}=\mathrm{knot}, \mathrm{S}=$ suture. 


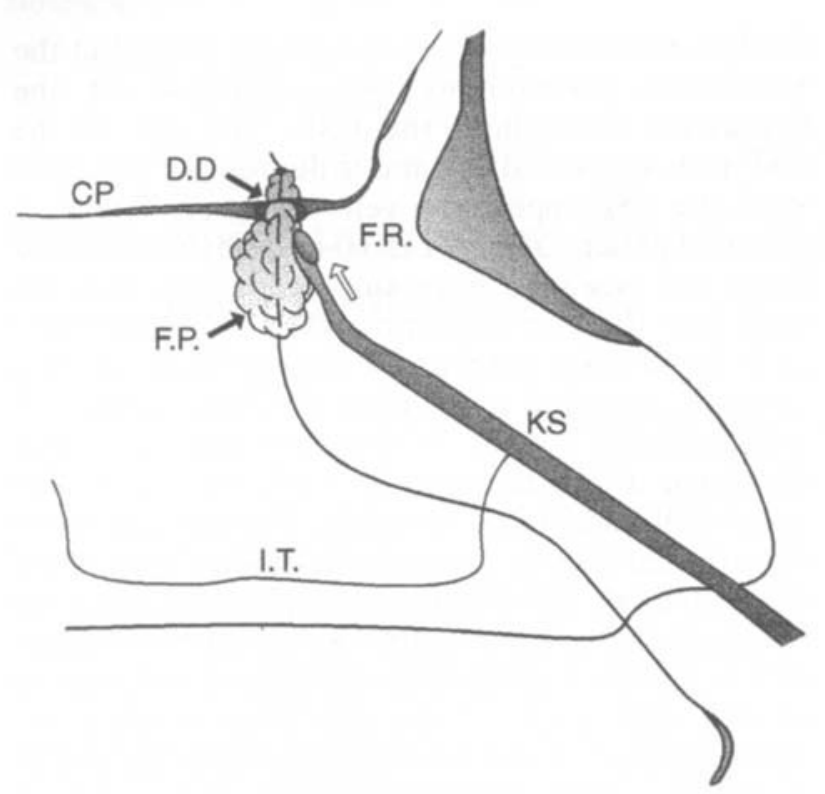

FIG. 3

The fat plug and suture are gently manipulated through the dural defect by blunt instruments Key: $\mathrm{CP}=$ cribriform plate, $\mathrm{DD}=$ dural defect, $\mathrm{FP}=$ fat plug, $\mathrm{FR}=$ frontal recess,

$\mathrm{IT}=$ inferior turbinate, $\mathrm{KS}=$ Kuhn spoon, $\mathrm{S}=$ suture.

the entrance of the dural defect. A fine Kuhn spoon or duckbill elevator (from the ear instrument tray) was used to gently push the entire fat plug through the dural defect into the intra-cranial cavity (Figure 3). Gentle traction on the vicryl suture caused the fat to concertina and widen and solidly seal the dural defect (Figure 4). No further CSF drainage was visible after traction was applied to the suture. Tisseal ${ }^{\circledR}$ glue was placed on the dural defect and the free mucosal graft. The vicryl suture was placed through the free mucosal graft and the graft slid up the suture (Figure 5) and placed over the bony skull base defect that had been previously cleared of mucosa. Similarly the suture was placed

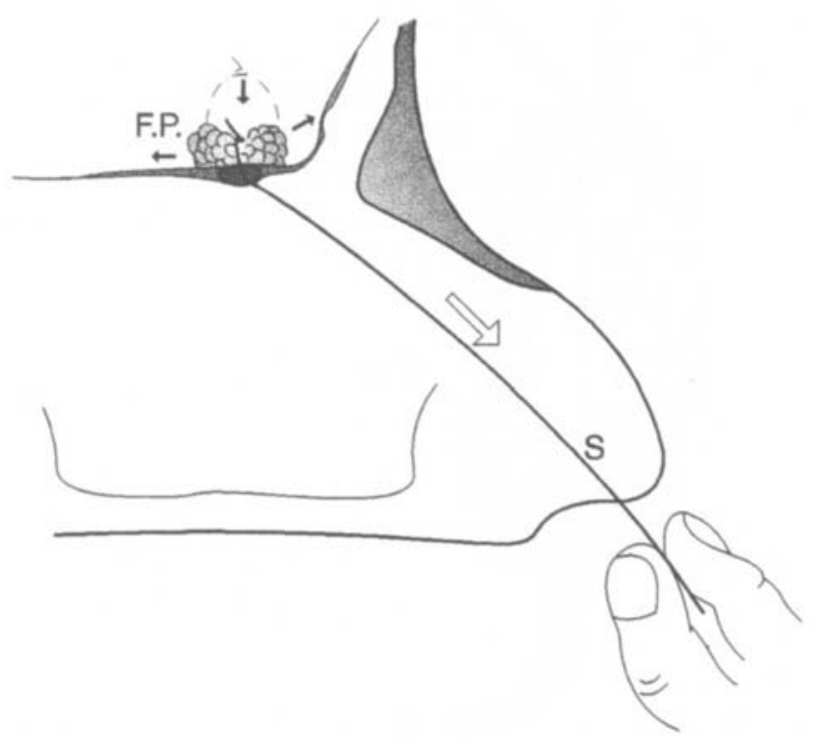

FIG. 4

Traction is placed on the vicryl suture causing expansion of the fat plug intracranially and sealing the dural defect like a plug sealing the bath. Key: FP = fat plug, $S=$ suture.

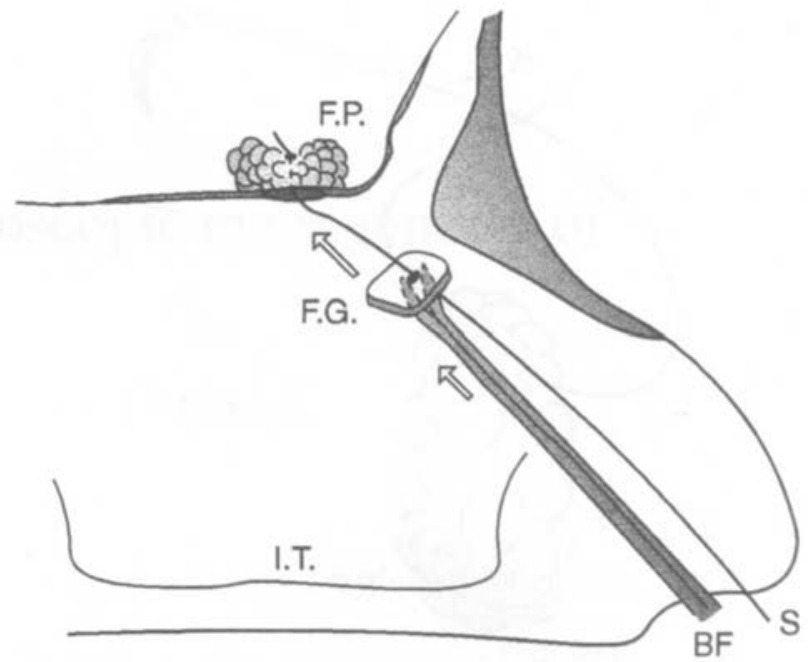

FIG. 5

The suture is placed through the free mucosa graft and it is pushed up the suture to cover the mucosal skull base defect. Key: BF = Blakelsey forceps, FG = free graft, FP = fat plug, $\mathrm{IT}=$ inferior turbinate, $\mathrm{S}=$ suture.

through an appropriately cut piece of Gelfoam ${ }^{\circledR}$ soaked in Tisseal ${ }^{\circledR}$ which was placed over the graft (Figure 6). The suture was then placed through the upper lateral cartilage of the nose, the tension adjusted and the suture taped onto the skin of the dorsal surface of the nose (Figure 6).

Finally a large piece of Gelfoam ${ }^{\circledR}$ was glued over the grafted area leaving the inferior nasal airway patent. The patients were extubated before full recovery to avoid coughing and straining on the endotracheal tube. The lumbar drain was left on free drainage $10 \mathrm{~cm}$ above the level of the heart with the patient in $15^{\circ}$ head up to prevent air being siphoned into the intrathecal space. This arrangement allows CSF to drain only if the CSF pressure is elevated. The lumbar drain was removed after 24 to 72 hours and the patient slowly mobilized. The vicryl suture

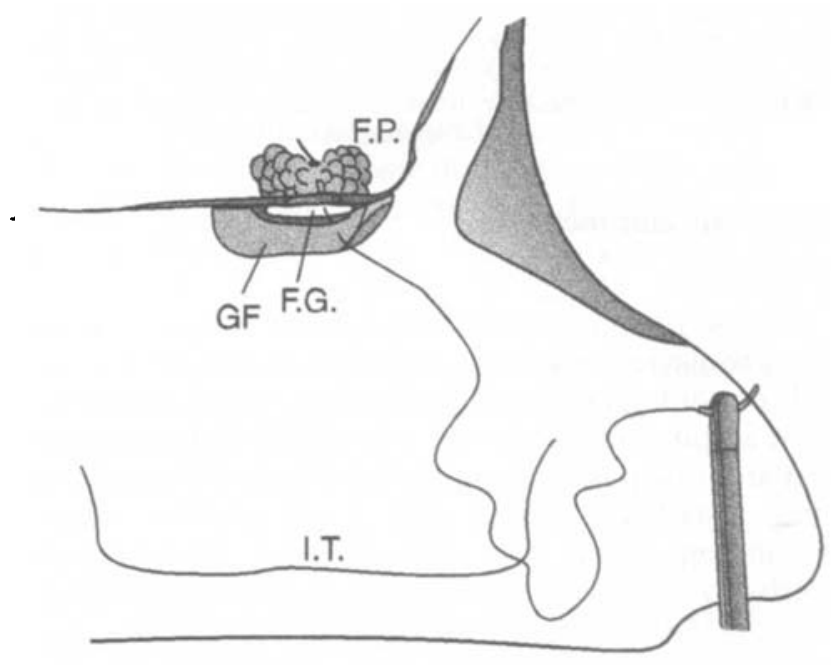

FIG. 6

Gelfoam ${ }^{\circledast}$ is placed over the mucosal graft and the suture is passed through the upper lateral nasal cartilage and secured to the nasal dorsum with a Steristrip ${ }^{\star}$ Key: FG $=$ free graft, $\mathbf{F P}=$ fat plug, $\mathrm{GF}=$ Gelfoam $^{\otimes}, \mathrm{S}=$ suture, $\mathrm{IT}=$ inferior turbinate. 
TABLE I

THE AETIOLOGY, SIZE AND SITE OF FISTULA, TREATMENT AND OUTCOME FOR THE PATIENTS TREATED WITH THE BATH PLUG TECHNIQUE

\begin{tabular}{|c|c|c|c|c|c|c|c|c|c|}
\hline No. & Age & Sex & Aetiology & $\begin{array}{l}\text { Size in } \\
\mathrm{mm}\end{array}$ & Site & $\begin{array}{l}\text { Lumbar } \\
\text { drain }\end{array}$ & Flu. & $\begin{array}{l}\text { Follow- } \\
\text { up }\end{array}$ & Result \\
\hline 1 & 44 & $\mathrm{~F}$ & Meningo-encephalocoele & 13 & $\begin{array}{l}\text { Left anterior fovea ethmoidalis } \\
\text { just posterior to AEA }\end{array}$ & Yes & Yes & $\begin{array}{c}18 \\
\text { months }\end{array}$ & Success \\
\hline 2 & 48 & $\mathrm{~F}$ & Spontaneous leak & 5 & $\begin{array}{l}\text { Right anterior cribriform plate } \\
\text { adjacent to AEA }\end{array}$ & Yes & Yes & $\begin{array}{c}17 \\
\text { months }\end{array}$ & Success \\
\hline 3 & 5 & F & Meningo-encephalocoele & 14 & $\begin{array}{l}\text { Left anterior cribriform plate } \\
\text { adjacent to AEA }\end{array}$ & Yes & No & $\begin{array}{c}3 \\
\text { months }\end{array}$ & Success \\
\hline 4 & 28 & F & Meningioma post-radiotherapy & 12 & Roof of the sphenoid - right side & Yes & Yes & $\begin{array}{c}18 \\
\text { months }\end{array}$ & Success \\
\hline 5 & 31 & F & Trauma & 3 & $\begin{array}{l}\text { Right lateral wall of olfactory fossa } \\
\text { adjacent to AEA }\end{array}$ & Yes & Yes & $\begin{array}{c}8 \\
\text { months }\end{array}$ & Success \\
\hline 6 & 62 & F & Spontaneous leak & 8 & $\begin{array}{l}\text { Left posterior ethmoid at junction } \\
\text { of superior turbinate to fovea } \\
\text { ethmoidalis }\end{array}$ & Yes & Yes & $\begin{array}{c}14 \\
\text { months }\end{array}$ & Success \\
\hline
\end{tabular}

Key: $\mathrm{AEA}=$ anterior ethmoidal artery, Flu. $=$ fluorescein.

was cut flush with the skin on day 5. The patients were advised to gently douche their nose three times a day with sterile saline and were reviewed weekly until the Gelfoam ${ }^{\circledR}$ had spontaneously resorbed

\section{Patients}

Since devising and testing this technique on cadavers two years ago, the authors have used it on all consecutive patients presenting with a CSF leak. Seven patients have presented with CSF leaks. Six patients have had the CSF leak endoscopically repaired using the 'bath-plug technique'. The patient details and aetiology of the leaks are summarized in Table I. In one patient the CSF leak could not be pre-operatively identified on CT scanning. The patient underwent lumbar drain insertion with fluorescein instillation and localization of the leak was attempted endoscopically. Unfortunately the leak could not be endoscopically identified despite Trendelenberg positioning. This patient then underwent a craniotomy and intradural closure of the leak. A $1 \times 0.5 \mathrm{~mm}$ leak was found at craniotomy in the anterior cribriform plate. This was successfully closed with a pericranial fascial graft. In retrospect if the leak had been found at endoscopy, it would have been suitable for closure using the 'bath-plug technique'.

\section{Results}

The exact locations and sizes of the leaks are summarized in Table I. All six patients underwent a bath-plug repair of the CSF leak with five receiving intra-thecal fluorescein. All patients had peri-operative antibiotic cover. All six patients had an uneventful post-operative recovery and none have shown any evidence of a recurrence of the leak. The follow-up period varies from three to 18 months with a mean of 13 months.

There were no complications from the fluorescein or lumbar drain and the CSF microscopy and culture were negative after lumbar drain removal. The patients were reviewed weekly as outpatients and performed sterile saline nasal douches until the Gelfoam $^{\circledR}$ had resorbed. The Gelfoam ${ }^{\circledR}$ took between three to five weeks to resorb. The mucosal graft over the defect could then be clearly visualized and was vascularized and healthy.

\section{Discussion}

One of the problems with endoscopic closure of CSF leaks has been that the graft is placed on the undersurface of the bony defect. It may not be in contact with the dural defect and the pressure from the CSF tends to push the graft away from the skull base. The authors believe that the 'bath-plug technique' overcomes both these problems as the plug is placed in the dural defect and the seal is maintained by the CSF pressure.

As the number of patients in this series is small, this technique cannot be said to be superior to any other technique although it does follow sound physical principles. Although the technique is simple, the introduction of the vicryl-fat plug intra-cranially is potentially problematic. If the fat plug is too big (wide), the plug will not fit through the dural tear and if force is used, the tear may be enlarged or if the dura suddenly gives way, intra-cranial damage may occur. Damage could occur to the olfactory nerve, anterior cerebral artery and frontal lobe. The authors advocate that force should never be used and that the plug be trimmed if it does not fit through with gentle manipulation using fine but blunt instruments. If the plug is too small (narrow) or too short, the plug will extrude when traction is placed on the vicryl suture. A custom-made fat inserter is being developed to overcome these problems.

Plug expansion occurs due to traction along the length of the plug with broadening of the plug as it is squashed from top to bottom (Figure 4). This technique was adequate for patients in this series in whom the dural defects were less than $15 \mathrm{~mm}$. If however, the defect is larger than this, bony support of the fat plug may be necessary. Preliminary cadaver work using the middle turbinate bone as an additional support has been done. The bone is fashioned so that it is narrower than the skull base defect but longer by about $3 \mathrm{~mm}$. 
The dura is elevated from the edges of the bony defect (difficult on the cribriform plate) for 3-4 mm with a blunt right-angled hook from the ear instrument tray. The cutting four 0 vicryl needle should be pushed through the centre of the bone and the hole slightly enlarged with the needle. The bone is slid between the dura and skull base in one direction until all the bone is intra-cranial and then using the suture should be slid in the opposite direction between the dura and skull base thus supporting the fat plug.

Two other aspects of our management are of importance. These are the use of a lumbar drain and intrathecal fluorescein. Some authors state that lumbar drains are unnecessary and have the added risk of sepsis and may allow air to be siphoned intracranially (Dodson et al., 1994; Hughes et al., 1997). Others maintain it is useful in patients with longstanding CSF leaks as the production of CSF is increased in these patients to compensate for the ongoing CSF loss. This may lead to high CSF pressures being generated post-operatively with an increased risk of failure (Mattox and Kennedy, 1990; Stankiewicz, 1991; Stankiewicz, 1995). Although CSF pressure improves the seal with the 'bath plug technique', theoretically excessive CSF pressure may push the plug through the defect especially if the plug is too small. Consequently we recommend the use of the lumbar drain for a few days in an attempt to maintain a normal CSF pressure.

As regards the use of fluorescein, complications including meningeal irritation, numbness and weakness of the lower extremities and seizures have been described (Papay et al., 1989; Mattox and Kennedy, 1990). However, if the lowest possible concentration is used (as in this series), the incidence of complications is rare and the complications are reversible (Mattox and Kennedy, 1990). If complications do occur, Moseley et al. (1978) recommended irrigation of the lumbar intra-thecal space with saline and elevation of the patient's head to confine the irritation to the lower limbs. The concentration used in this series of patients stained the CSF a bright green-yellow and special light sources or filters were not required to positively identify the site of the fistula. In this series no complications were seen from intra-thecal fluorescein used in the low doses described.

One of the critical aspects of endoscopic closure of CSF leaks is the identification of the site of the leak. This should, if possible, be done pre-operatively with high resolution CT scanning, MRI scanning, metrizamide CT scanning or radio-isotope scanning. Metrizamide is a water-soluble contrast media that is injected intra-thecally and if a CSF leak is present, this should be seen on CT scanning (Manelfe et al., 1982). Once the general area of the leak has been identified, exact location is done at the time of surgery. Useful adjuvants are intra-thecal fluorescein, placing the patient in the Trendelberg position, performing Valsalva manoeuvres and once the lumbar drain has been placed, injecting 100 to $200 \mathrm{ml}$ of saline intra-thecally (Papay et al., 1989). As CSF leaks are a significant complication of endoscopic sinus surgery, surgeons should be aware of the techniques available for CSF leak repair. The 'bath-plug' technique is simple and suited to treating these surgically created skull base defects. This includes CSF leaks of a similar size found or surgically created in the attic or mastoid of the ear. However, this technique is not suitable for the closure of long linear fractures of the skull base. A technique using similar principles is being developed for these fractures.

\section{References}

Aarabi, B., Leibrock, L. (1992) Neurosurgical approaches to cerebrospinal fluid rhinorrhea. Ear, Nose and Throat Journal 71: $300-305$.

Carmel, P., Komisar, A. (1991) Cerebrospinal fiuid rhinorrhea. In Surgery of the Paranasal Sinuses (Blitzer, A., Lawson, W., Friedman, W., eds.). W. B. Saunders Company, Philadelphia, pp 377-388.

Dodson, E., Gross, C., Swerdloff, J., Gustafson, M. (1994) Transnasal endoscopic repair of cerebrospinal fluid rhinorrhoea and skull base defects: A review of twentynine cases. Otolaryngology - Head and Neck Surgery 111: $600-605$.

Hisamatsu, K., Ganbo, T. (1995) Spontaneous cerebrospinal fluid rhinorrhoea through the cribriform plate fistula cured by endonasal surgery: transeptal submucoperiosteal obliteration of the olfactory cleft. Otolaryngology - Head and Neck Surgery 113: 822-825.

Hoa, S. (1996) Transnasal endoscopic repair of cerebrospinal fluid rhinorrhoea: an interposition technique. Laryngoscope 106: 501-503.

Hughes, R., Jones, N. S., Robertson, I. (1997) The endoscopic treatment of cerebrospinal fluid rhinorrhoea: the Nottingham experience. Journal of Laryngology and Otology 111: 125-128.

Lund, V. J., MacKay, I. S. (1994) Outcome assessment of endoscopic sinus surgery. Journal of the Royal Society of Medicine 87: 70-72.

Manelfe, C., Cellerier, P., Sobel, D., Prevost, C., Bonafé, A. (1982) Cerebrospinal fluid rhinorrhoea: Evaluation with Metrizamide cisternography. American Journal of Roentgenology 138: 471-476.

Mattox, D., Kennedy, D. (1990) Endoscopic management of cerebrospinal fluid leaks and cephalocoeles. Laryngoscope 100: $857-862$.

Moseley, J., Carton, C., Stern, W. (1978) Spectrum of complications in the use of intrathecal Fluorescin. Journal of Neurosurgery 48: 765-767.

Papay, F., Maggiano, H., Dominquez, S., Hassenbusch, S., Levine, H., Lavertu, P. (1989) Rigid endoscopic repair of paranasal sinus cerebrospinal fluid fistula. Laryngoscope 99: 1195-1201.

Stankiewicz, J. (1991) Cerebrospinal fluid fistula and endoscopic sinus surgery. Laryngoscope 101: 250-256.

Stankiewicz, J. (1995) Cerebrospinal fluid fistula and endoscopic sinus surgery. In Advanced Endoscopic Sinus Surgery (Stankiewicz, J., ed.). Mosby, St Louis, pp 81-86.

Address for correspondence:

P. J. Wormald,

Department of Surgery,

Prince of Wales Hospital,

Shatin, NT,

Hong Kong. 